\title{
A Real-time Implementation of an Intersection Collision Avoidance System *
}

\author{
Mattias Brännström* Jonas Sjöberg ${ }^{* *}$ Linus Helgesson ${ }^{* *}$ \\ Mikael Christiansson** \\ * Volvo Car Corporation, Department of Active Safety and Chassis, \\ Gothenburg, Sweden (e-mail: mbranns3@volvocars.com) \\ ** Chalmers University of Technology, Department of Signals and \\ Systems, Gothenburg, Sweden (e-mail: jonas.sjoberg@chalmers.se)
}

\begin{abstract}
:
This paper presents a real-time implementation of a collision avoidance (CA) system that uses autonomous braking and model predictive control to assist drivers in avoiding collisions with other road users. To the authors knowledge, this is the first CA system that targets general vehicle collisions that has been implemented in a car. The system is based on a recently published decision-making algorithm which is described in [1]. To validate the CA system in various collision scenarios without endangering the driver of the vehicle, a novel test platform has been developed. The test platform consist of a soft crashable obstacle which is movable in speeds up to $70 \mathrm{~km} / \mathrm{h}$ and safe to collide with in any angle in relative speeds up to $100 \mathrm{~km} / \mathrm{h}$. In the current implementation, estimates of the motion of the obstacle are obtained through a reference sensor fusion system that is based on a combination of in-vehicle sensors and a differential global positioning system. Results from both intersection and rear-end collision situations are presented. The results show that the proposed CA system can be implemented in a real-time environment and that the predictive brake control algorithm accurately accounts for delays and ramp-up times in the brake system of the vehicle.
\end{abstract}

Keywords: Road traffic; Obstacle avoidance; Safety; Active brake control; Model-based control; Autonomous control; Real-time systems

\section{INTRODUCTION}

Collisions between vehicles and other road user cause millions of serious injuries and fatalities every year [2]. In Sweden, it is estimated that as many as one out of thirty citizens becomes seriously injured in road traffic accidents at some point in life [3]. A vision, shared by the Swedish Road Administration and the Volvo Car Corporation, is that no one shall be killed or seriously injured in road traffic accidents [4]. As a part of this vision, novel systems for assisting drivers in avoiding or mitigating collisions are continuously being introduced to the market [5].

Previous collision avoidance (CA) systems that have been implemented in real-time systems are restricted to specific collision types, such as rear-end collisions or collisions with pedestrians that cross the road in front of the vehicle. However, there is a wide variety of collision scenarios in real traffic accidents, especially in intersection environments. Examples of common collision types are illustrated in Fig. 1. Clearly, there is a need for CA systems that target a larger class of collision scenarios than only rearend collisions and collisions with pedestrians.

\footnotetext{
^ This work was supported in part by the Intelligent Vehicle Safety Systems programme of the Swedish National Road Authorities and in part by the Volvo Car Corporation.
}

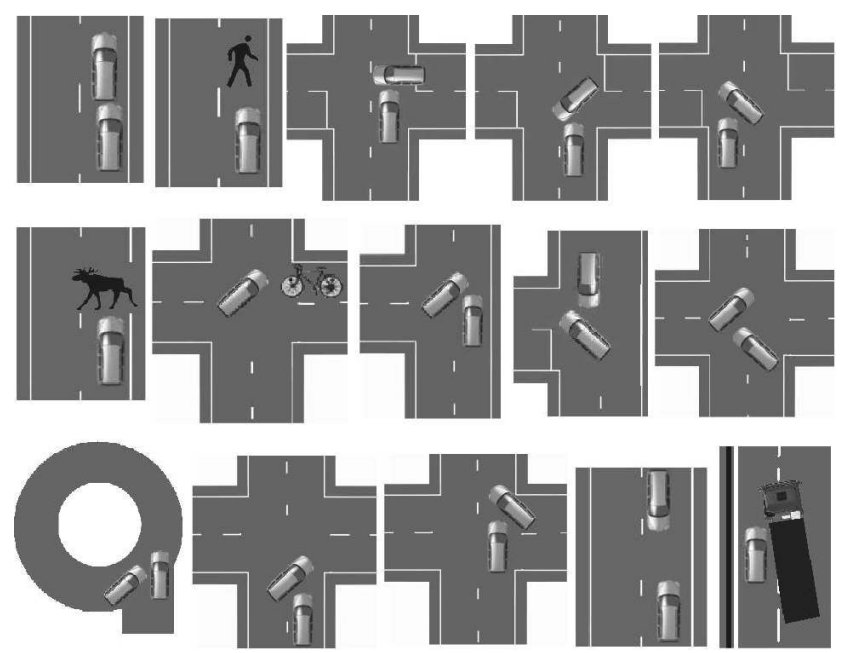

Fig. 1. Examples of collision scenarios with various objects.

This paper presents a real-time implementation of a CA system that targets all types of collisions in all types of traffic scenarios. The CA system and its implementation in a Volvo V70 is described in Section 2. A novel test platform for validating the $\mathrm{CA}$ system in critical traffic situations without endangering the driver is presented in Section 3. Results from both an intersection collision scenario and a rear-end collision scenario are presented in Section 4. Finally, conclusions are drawn in Section 5. 


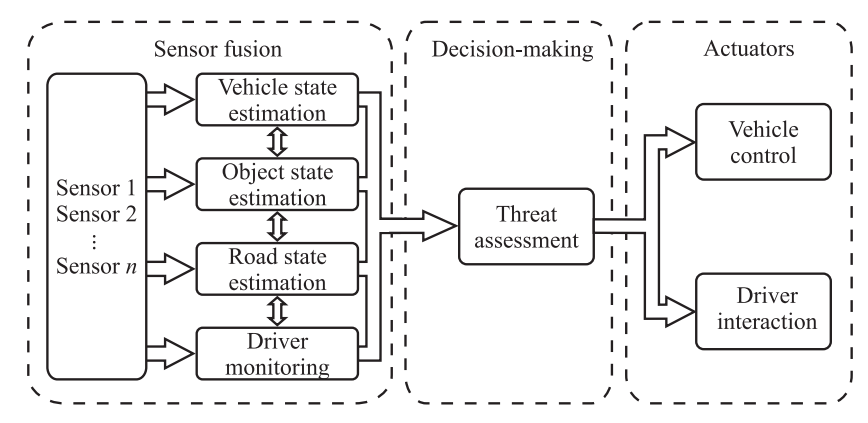

Fig. 2. Collision avoidance systems consist of three layers.

\section{THE COLLISION AVOIDANCE SYSTEM}

This section gives a short introduction to the decisionmaking algorithm, the brake controller and the sensor system that are used in the current implementation. The decision-making algorithm is described in detail in [1].

\subsection{System components}

Collision avoidance systems can generally be divided into three layers, as illustrated in Fig. 2.

- A sensor fusion system collects and fuses information from sensors to estimate the motion and properties of surrounding road users and objects.

- A decision-making algorithm uses the estimates to assess the traffic situation and make decisions on when and how to assist the driver.

- Finally, an actuator system warns the driver or influences the motion of the vehicle through autonomous brake or steer interventions.

The objective with all types of CA systems is to assist drivers in avoiding or mitigating collisions, without disturbing the driver with unnecessary warnings or interventions. The latter statement is important to gain high customer acceptance for these types of systems. It also makes a clear cut distinction between CA systems and systems for fully autonomous driving applications which control the vehicle at all times. The present paper focuses on CA systems that use autonomous braking to assist drivers in avoiding collisions. Other countermeasures, such as warnings and autonomous steering are not discussed.

\subsection{Decision-making and brake control}

The decision-making algorithm makes predictions of the future trajectories of other road users, as illustrated in Fig. 3, and uses a model predictive control (MPC) approach to obtain analytical solutions on how the driver of the vehicle can either steer, brake or accelerate to avoid a collision during a limited prediction horizon [1]. The algorithm minimizes the fraction of the estimated tire-toroad friction that needs to be utilized to avoid a collision. An introduction to the MPC concept is given in [6].

In order to maintain driver autonomy, the CA system is designed to initiate autonomous braking when it is estimated that the driver no longer can avoid a collision by steering or by accelerating and that the driver needs to use at least $80 \%$ of the estimated tire-to-road friction to avoid a collision by braking. To further reduce the risk of

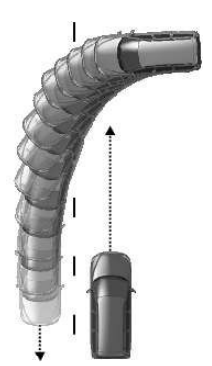

Fig. 3. The host vehicle (bottom) is approaching another vehicle (top). The objective is to avoid a collision during the entire prediction horizon.

unnecessary braking, which may disturb the driver, brake interventions are inhibited if it is estimated that another road user can manoeuvre to avoid an impending collision either by braking, steering or accelerating.

During brake interventions, the MPC algorithm assesses the traffic situation in real-time and outputs the required deceleration profile on the car's Controller Area Network (CAN-bus) to a brake control module. The autonomous brake system consists of brake pads and a brake pump that can decelerate the car with up to $-10 \mathrm{~m} / \mathrm{s}^{2}$ at a maximum rate of $-20 \mathrm{~m} / \mathrm{s}^{3}$, after an initial internal delay of approximately $150 \mathrm{~ms}$. Since the traffic situation may change during the intervention, the required deceleration to avoid a collision is estimated by the algorithm at a rate of $40 \mathrm{~Hz}$ and the brakes are controlled throughout the intervention. The algorithm accounts for both time delays and ramp-up times in the actual brake system when the brake control signal is updated [1].

In the presence of measurement and prediction uncertainties, the brake control signal may be selected e.g. by propagating the uncertainties through the MPC algorithm or by using monotonicity properties to estimate the required deceleration for the "worst-case-prediction" of the obstacle's future path, thus allowing the system to avoid a collision with some safety margin. However, in this paper it is not further investigated how different measurement and prediction uncertainties may affect the brake control.

\subsection{The reference sensor system}

In the current implementation, a reference sensor fusion system is used to obtain estimates of the motion and properties of surrounding road users. The basic principle behind the reference system is to let a differential global positioning system (DGPS) keep track of the position of the host vehicle and then let one or several crashable obstacles communicate their global positions to the host vehicle via wireless local area network (WLAN) communication. The relative position, velocity and heading angle between the host vehicle and each obstacle can then be estimated by the host vehicle. Note that this sensor system is not intended for production vehicles. Rather, the purpose of using a reference sensor fusion system is to enable development and validation of all other key components in the CA system before suitable production ready sensors, such as radars and cameras, are available. This work process is used to accelerate the development of CA systems and to improve the quality of other key components as well as improving the complete CA system. 


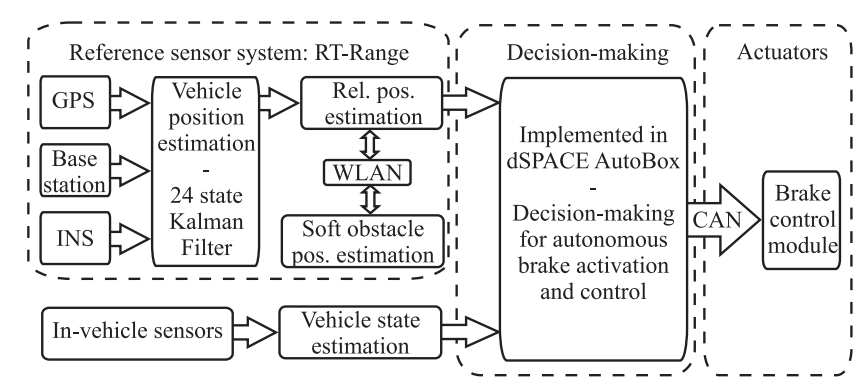

Fig. 4. Signal routing in the implemented CA system.

DGPS is an enhancement to the more commonly known global positioning system (GPS). By introducing a groundfixed reference station that broadcasts the difference between the positions indicated by the GPS system and the known fixed position of the reference station, a higher positioning accuracy can be achieved. To maintain a high accuracy even in dynamic driving situations, a Kalman filter with 24 states is updated at a sample rate of $100 \mathrm{~Hz}$ using measurements from the DGPS in combination with an inertial navigation system (INS) with three highly accurate angular rate sensors (gyroscopes) as well as three accelerometers. This reference system has been developed by Oxford Technical Solutions and is called RT-Range, which has a positioning accuracy of a few centimeters [7].

The global positions of the crashable obstacles are obtained through a test platform that controls the motion of the obstacles. The obstacle can either be controlled by an accurate wire system that keeps track of the obstacle's global position, or it can be hung by the side of another vehicle that is also equipped with a DGPS system. In this paper, a wire system with a positioning accuracy of a few centimeters is used to control the motion of the obstacle. The obstacle and the wire system are later described in more detail in Section 3.

The estimates from the reference sensor fusion system along with measurements of the host vehicle speed, steering angle, etc. from standard in-vehicle sensors are sent to the decision-making algorithm, which has been implemented in a dSPACE AutoBox that has been installed in a Volvo V70. The system components and the signal routing are illustrated in Fig. 4.

\section{THE TEST PLATFORM}

This section provides an overview of different methods for validating CA systems and a description of the test platform that is proposed and used in this paper.

\subsection{Methods for validating CA systems}

There are many complementary methods for validating CA systems. Schöner et al. give an overview of different methods in [8], where a test method that uses coordinated automated driving robots in combination with a DGPS system is proposed for validating CA systems in near crash situations. Examples of other methods are driving simulators and computer simulations, which are useful especially in early phases of the development process [9]. Closer to the production phase, there is a need for validation in

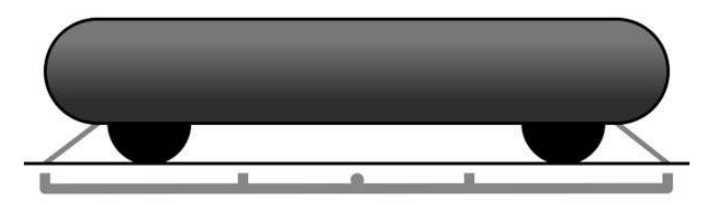

Fig. 5. The soft car is attached to a trolley.

real vehicles with real drivers in critical traffic situations, without endangering the driver of the vehicle. Such tests are essential since the objective with CA systems is to actually avoid collisions without disturbing the driver of the vehicle. Since collisions may also be avoided by the driver without using assistance systems, it is the driver that has to judge if a warning or an intervention was justified or not. In practice, this means that it is necessary to have drivers present when validating CA systems in near-crash scenarios, at least until suitable driver models with objective performance measures have been developed. Thus, the development process cannot be completed solely through off-line testing or by using automated driving robots, even if such methods are very useful.

One method for safely validating CA systems in near crash and crash situations with a driver present in the vehicle is to use soft crashable obstacles in some form [10]. Some of these soft obstacles are designed to quickly be removed from the driving path just prior to a collision to reduce wear and tear, but the obstacles are always designed to be safe to collide with. However, since the object is safe to collide with, soft obstacles are not always optimal when validating that the $\mathrm{CA}$ system does not disturb the driver with unnecessary braking. The reason for this is that the driver knows that the obstacle is soft and that a collision will not hurt. The driver can without any sensation of fear pass really close to the soft obstacle at high relative speeds, which may not be the case if driving towards a real vehicle or a pedestrian. Thus, to validate the CA system with respect to unnecessary interventions, extensive field operational tests with different types of drivers in various types real traffic conditions is a natural complement to the aforementioned validation methods [11].

Many previous CA systems have been developed partly by using soft crashable dummies or inflatable cars in near crash and collision situations [12]. Autoliv among others has previously proposed soft crashable cars that can be used in intersections collision situations, but these test platforms have been restricted to collision situations in the speed range for city driving [13].

\subsection{The proposed test platform}

In this subsection, a new test platform for validating CA systems in intersection collision scenarios is presented. The developed platform consists of a soft crashable car that is movable along a $400 \mathrm{~m}$ long track in speeds up to $70 \mathrm{~km} / \mathrm{h}$ and crashable from any angle in relative speeds up to $100 \mathrm{~km} / \mathrm{h}$. The soft car, which weights approximately $10 \mathrm{~kg}$ and has the size of a small sports car $(3.7 \times 1.6 \mathrm{~m})$, is attached to a trolley which is driven by a wire system. The entire trolley is descended a few centimeters into the roadway, as shown in Fig. 5. 


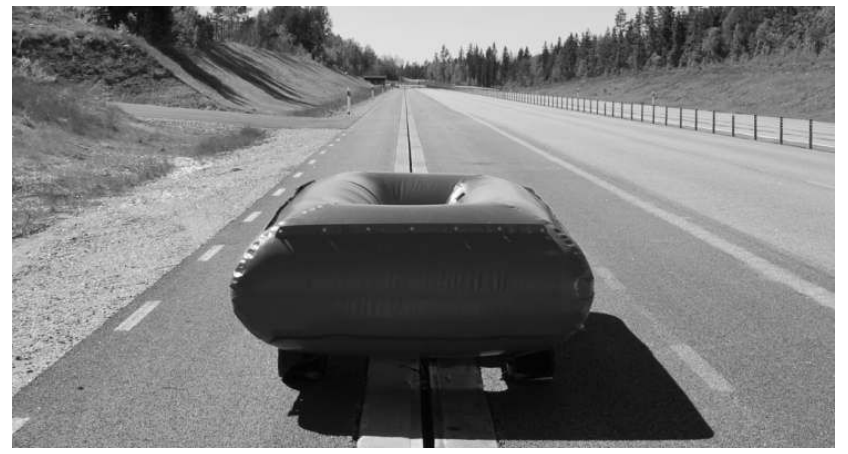

Fig. 6. The test track with the soft crashable car.

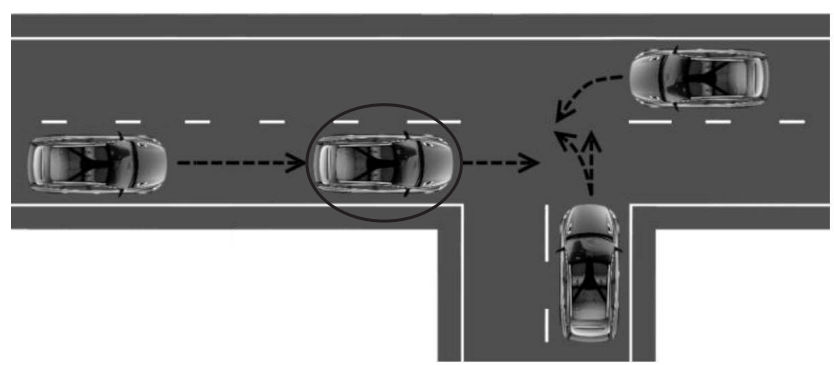

Fig. 7. Examples of collision scenarios that can be validated with the test platform. The soft car is encircled.

If the soft car is struck by the test vehicle from any angle, it is mechanically detached from the trolley, which stays hidden in the track. The test platform is restricted to intersection and rear-end collision scenarios where the soft car is traveling on a straight road, but the principal construction may be extended to cover an even larger range of collision scenarios. The test track and the soft car are shown in Fig. 6.

The wire system keeps track of the the trolley's position, velocity and acceleration and thus also the soft car's position. These estimates are continuously communicated to the sensor reference system in the host vehicle via WLAN, as previously illustrated in Fig. 4 and described in Section 2.

The soft car is equipped with radar reflective material, such that the platform can also be used to validate CA systems with e.g. radar or lidar sensors intended for production vehicles. To validate CA systems that use cameras in the sensor fusion layer, the soft car can also be equipped with an upper part and a cover to resemble different vehicle shapes and colors.

In order to validate the CA system in different types of intersection collision situations, a T-junction has been constructed along the track, as can be seen in the top left corner of Fig. 6. Examples of collision scenarios that can be validated in the test platform are illustrated in Fig. 7.

\section{RESULTS}

This section presents results from tests where the CA system is validated in a rear-end collision scenario and an intersection collision scenario. The scenarios are described and illustrated, followed by an analysis of the performance of the CA system in each scenario.

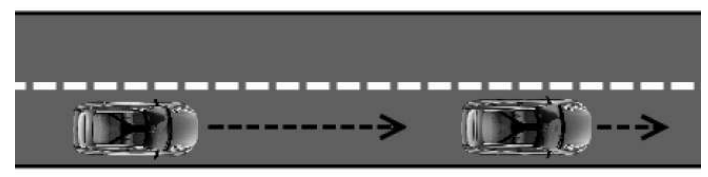

Fig. 8. Rear-end collision scenario, where the soft car is the lead vehicle.

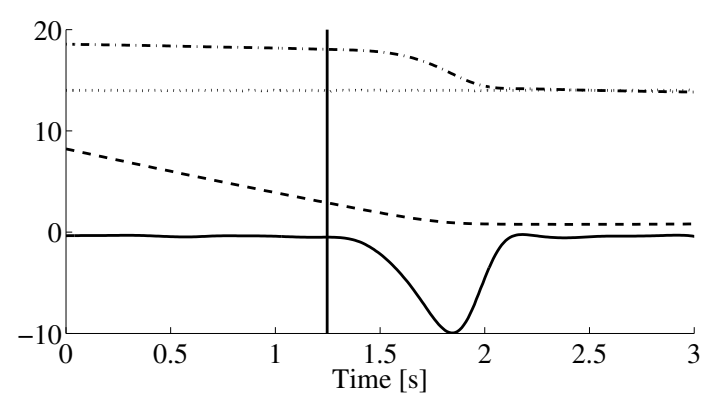

Fig. 9. Results from a rear-end collision scenario. The acceleration (solid line) and velocity (dash-dotted) of the host vehicle are shown along with the velocity of the soft car (dotted), all in SI-units. The dashed line shows the range between the two vehicles. The vertical line shows when the CA system initiates an autonomous brake intervention, that is when it is judged that a hard braking maneuver is the only option that is left to avoid a collision.

\subsection{Rear-end collision scenario}

In Fig. 8, a rear-end collision scenario is illustrated and the output from the CA system is shown in Fig. 9. In this scenario, the soft car is a lead vehicle which is driving at a constant speed of $14 \mathrm{~m} / \mathrm{s}(50 \mathrm{~km} / \mathrm{h})$. The vehicle hosting the CA system has an initial speed of $19 \mathrm{~m} / \mathrm{s}(68 \mathrm{~km} / \mathrm{h})$. In Fig. 9, it can be noticed that there is an initial time delay of about $150 \mathrm{~ms}$ in the brake actuation and that the deceleration change rate is approximately constant in the time interval $1.45-1.85 \mathrm{~s}$. Note that the range between the vehicles directly after the intervention is less than $1 \mathrm{~m}$ and that the vehicles are traveling at approximately the same velocity. Even though a collision was avoided by the CA system, which performed exactly as expected based on off-line simulations, it may be uncomfortable for the driver to stay this close to the lead vehicle at high speed for an extended period of time. It may be desirable in future CA systems to continue the brake intervention until e.g. a suitable time gap to the lead vehicle has been obtained.

Since the dynamics of the brake system are modeled in the decision-making algorithm [1], the deceleration profile in Fig. 9 becomes consistent and smooth. During the intervention, the required deceleration to avoid a collision is reevaluated every $25 \mathrm{~ms}$ and the deceleration request to brake system is adjusted accordingly. This makes the CA system robust to modeling errors and the system is adapted to possible changes that may occur in the traffic situation during the brake intervention. The brakes are released when it is estimated that no additional deceleration is required to avoid a collision. 


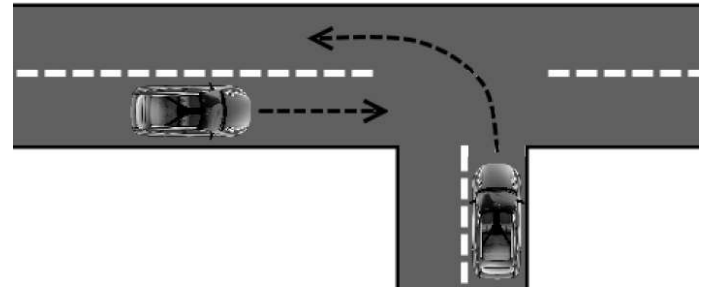

Fig. 10. Left turn across path - lateral direction scenario (LTAP-LD), where the soft car is the vehicle illustrated to the left.

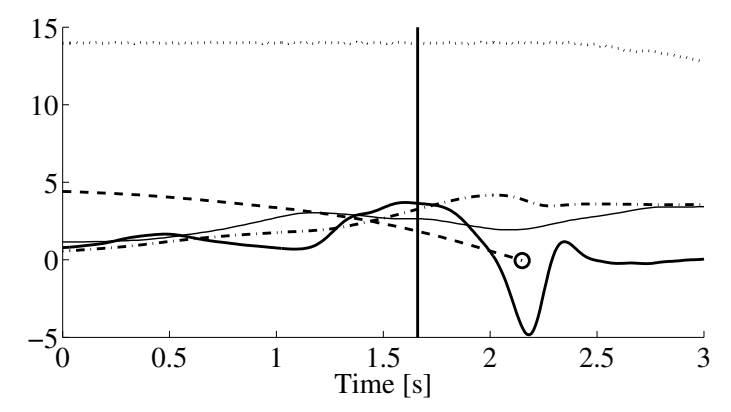

Fig. 11. Results from a LTAP-LD scenario. The acceleration (solid line) and velocity (dash-dotted) of the host vehicle are shown along with the velocity of the soft car (dotted), all in SI-units. The steering wheel angle (thin solid line) is given in radians and defined as positive when turning to the left. The vertical line shows when the brake intervention is initiated. The dashed line shows the range between the two vehicles in the longitudinal direction of the host vehicle.

\subsection{Intersection collision scenario}

In Fig. 10, an intersection collision scenario is illustrated and the output from the CA system is shown in Fig. 11. The soft car is traveling at a constant speed of $14 \mathrm{~m} / \mathrm{s}$ and the host vehicle accelerates from standstill to $4 \mathrm{~m} / \mathrm{s}$ while initiating a left turn onto a main road. The driver of the vehicle accelerates with $3.7 \mathrm{~m} / \mathrm{s}^{2}$ when the brake intervention is initiated at the time $1.6 \mathrm{~s}$. The engine torque is not reduced during brake interventions in the current implementation of the CA system, but the autonomous braking reduces the acceleration of the vehicle such that a collision is avoided.

At the time $2.15 \mathrm{~s}$, the soft car has passed the intersection and the vehicle with the CA system can pass behind the soft car. The range between the vehicles is encircled at this point in time. Once the soft car has passed the intersection the risk of a collision is non existent and thus the range is no longer displayed in the graph. The CA system avoided the collision by using a short brake intervention and then allowing the host vehicle to pass behind the crossing soft car. As in the rear-end collision scenario, the results are consistent with results from off-line simulations of similar traffic scenarios.

\section{CONCLUSIONS}

This paper shows that a proposed CA system for assisting drivers in avoiding arbitrary collisions with other road users can be implemented in a real-time application. Additionally, a novel test platform for validating CA systems in near-crash scenarios at intersections has been presented. The real-time implementation of the CA system has successfully been tested in rear-end and intersection collision scenarios. The results show that the CA system accurately can predict an impending collision and smoothly control the brakes of the vehicle such that a collision is avoided.

\section{REFERENCES}

[1] M. Brännström, E. Coelingh and J. Sjöberg. ModelBased Threat Assessment for Avoiding Arbitrary Vehicle Collisions. IEEE Trans. on Intelligent Transportation Systems, vol. 11, no. 3, pages 658-669, 2010.

[2] M. Peden, R. Scurfield, D. Sleet, D. Mohan, A. Hyder, E. Jarawan and C. Mathers. World report on road traffic injury prevention. World Health Organization, The World Bank, 2004.

[3] Road Traffic Injuries 2008. Swedish Institute for Transport and Communications Analysis, 2009.

[4] Management by objectives for road safety work Stakeholder collaboration towards new interim targets 2020. The Swedish Road Administration, 2008.

[5] E. Coelingh, A. Eidehall and M. Bengtsson. Collision Warning with Full Auto Brake and Pedestrian Detection - a practical example of Automatic Emergency Braking. 13th International IEEE Conference on Intelligent Transportation Systems, 2010.

[6] C. E. Garca, D. M. Prett and M. Morari. Model predictive control: Theory and practice - A survey. Automatica, vol. 25, 1989.

[7] RT-Range for ADAS testing. Fact sheet on "RTRange - Lane Position and Vehicle-to-vehicle Measurement", Oxford Technical Solutions, 2010.

[8] H.P. Schöner, S. Neads and N. Schretter. Testing and verification of active safety systems with coordinated automated driving. The 21th conference on Enhanced Safety of Vehicles, Paper Number 09-0187, 2009.

[9] M. Koashi, S. Hayakawa, T. Suzuki, S. Okuma, N. Tsuchida, M. Shimizu and S. Kido. Measurement and modeling of collision avoidance behavior of drivers using three dimensional driving simulator. SICE Annual Conference, vol. 1, pages 623-627, 2003.

[10] R. Kiefer, D. LeBlanc, M. Palmer, J. Salinger, R. Deering and M. Shulman. Development and validation of functional definitions and evaluation procedures for collision warning/avoidance systems. NHTSA Technical Report DOT HS 808 964, 1999.

[11] S. McLaughlin, J. Hankey and T. Dingus. A method for evaluating collision avoidance systems using naturalistic driving data. Accident Analysis and Prevention, vol. 40, pages 8-16, 2008.

[12] M. Avery and A. Weekes. Autonomous Braking Systems And Their Potential Effect On Whiplash Injury Reduction. The 21th conference on Enhanced Safety of Vehicles, Paper Number 09-0328, 2009.

[13] S-H. Nilsson. Carson City - A test track for urban envorenments. Swedish Automotive Testing Seminar, Fact sheet on "Carson City", 2010. 